\title{
Tecnologías de la Información y la Comunicación y el Conectivismo
}

\section{Information and Communication Technologies and Connectivism}

DOI: $10.46932 / \mathrm{sfjdv2n5-091}$

Received in: Oct 1st, 2021

Accepted in: Dec 30th, 2021

\section{Carlos Rios-Campos}

Investigador RENACYT

Doctor en Gestión Universitaria. Maestro en Administración Ingeniero de Sistemas

Docente de la Universidad Nacional Toribio Rodríguez de Mendoza de Amazonas

Miembro del Colegio de Ingenieros del Perú

Section Investigation Activities Chair North (IAC) - IEEE Perú. Bagua, Perú

E-mail: carlos.rios@untrm.edu.pe

\section{Verónica Vanessa Mackliff Peñafiel}

Magíster en Docencia y Currículo

Ingeniera en Sistemas

Docente del Instituto Superior Tecnológico Babahoyo

Babahoyo, Ecuador

E-mail: Veromackliff@gmail.com

\section{Freddy Manuel Camacho Delgado}

Docente Principal

Doctor en Economía

Universidad Nacional Intercultural Fabiola Salazar Leguía de Bagua

Bagua Grande, Perú

E-mail: fcamacho@unibagua.edu.pe

\section{Juan Alberto Avalos Hubeck}

Contador Público

Maestro en Tributación y Asesoría Fiscal

Universidad Nacional Toribio Rodríguez de Mendoza de Amazonas

E-mail: juanavalosh@hotmail.com

\section{Manuel Tiberio Valentín Puma}

Contador Público

Doctor en Gestión Pública

Magister en Administración de la Educación

Trabajo en la Universidad Nacional Intercultural Fabiola Salazar Leguía de Bagua Bagua, Perú

E-mail: mvalentin@unibagua.edu.pe

\section{Yesenia Paulina Valentín Huanaco}

Ingeniero pesqueiro

Trabajo en la Empresa Comunal Hatun Nan Pueblo Libre

E-mail: yeseniavalentin76@gmail.com 


\author{
Marco Antonio Odar Puse \\ Maestro en Ciencias Económicas \\ Mención en Gerencia Social. Economista \\ Grado de Bachiller en Ciencias Económicas \\ E-mail: marco_4009@hotmail.com \\ Enrique Guillermo Llontop Ynga \\ Médico Cirujano \\ Magister en Gestión de Servicios de Salud \\ Universidad Señor de Sipán \\ Universidad Cesar Vallejo \\ E-mail: ellontop@crece.uss.edu.pe
}

\begin{abstract}
RESUMEN
Resulta necesario conocer el estado de arte de las Tecnologías de la Información y la Comunicación y el conectivismo, considerando que a nivel mundial se presenta en la actualidad un desafío a la educación tradicional con el inicio de la pandemia en el año 2020. Se planteó el objetivo general, analizar las Tecnologías de la Información y la Comunicación y el conectivismo. Metodología, la investigación presentó un diseño cualitativo-interpretativo, de tipo documental, se han seleccionado 47 documentos, realizados en el periodo 2016 - 2021, incluyendo: artículos científicos, artículos de revision e información de sitios web de organizaciones reconocidas; a excepción de un documento del año 2005, de un autor referente en el tema. Las palabras claves utilizadas en las búsquedas fueron: Tecnologías de la Información y la Comunicación, conectivismo y educación. Resultados, el conectivismo como una teoría de aprendizaje de la nueva era digital, ayuda a mejorar el uso de las TIC, recordando que estas resultan herramientas muy importantes para los estudiantes de los diferentes niveles educativos. Conclusiones, las Tecnologías de la Información y la Comunicación y el conectivismo, son muy importantes en esta era digital, donde los nativos digitales tienen muchas ventajas en comparación a los migrantes digitales. En nuestros países latinoamericanos, la brecha digital limita la adopción de las TIC por las grandes mayorías.
\end{abstract}

Palabras clave: Tecnologías de la Información y la Comunicación, conectivismo y educación.

\begin{abstract}
It is necessary to know the state of the art of Information and Communication Technologies and connectivism, considering that at the global level there is currently a challenge to traditional education with the onset of the pandemic in 2020. The general objective, analyze Information and Communication Technologies and connectivism. Methodology, the research presented a qualitative-interpretative design, documentary type, 47 documents have been selected, made in the period 2016 - 2021, including: scientific articles, review articles and information from websites of recognized organizations; With the exception of a document from 2005, by a leading author on the subject. The keywords used in the searches were: Information and Communication Technologies, connectivism and education. Results, connectivism as a learning theory of the new digital age, helps to improve the use of ICT, remembering that these are very important tools for students at different educational levels. Conclusions, Information and Communication Technologies and connectivism are very important in this digital age, where digital natives have many advantages compared to digital migrants. In our Latin American countries, the digital divide limits the adoption of ICT by the great majority.
\end{abstract}

Keywords: Information and Communication Technologies, connectivism and education. 


\section{INTRODUCCIÓN}

Cuando a inicios de marzo fuera declarada pandemia por la OMS aún no se podían prever la magnitud de los daños humanos, económicos y sociales que traería la expansión de la enfermedad (Macias, 2020).

La enfermedad denominada COVID-19 es una pandemia causada por el virus SARS-CoV-2 (síndrome agudo respiratorio severo 2) (Córdova-Aguilar \& Rossani, 2020).

La COVID-19 ha dejado su devastadora estela, no solo de muerte, sino también ha puesto al descubierto las serias limitaciones de los sistemas sanitarios de los países. Esta develación se ha mostrado más devastadora en los países con marcadas deficiencias, pero sobre todo con extremas inequidades en sus determinantes sociales y económicas, como los de Latinoamérica (Cabezas, 2020).

Durante la pandemia la educación tradicional, ha sido desafiada y han surgido nuevas formas de interacción entre estudiantes y docentes. En la presente investigación se planteó el objetivo general, analizar las Tecnologías de la Información y la Comunicación y el Conectivismo.

En consecuencia, se estima que la comunidad internacional demanda de actuaciones educativas donde los estudiantes adquieran conocimientos y destrezas para la mejora de su competencia digital (Valverde, 2018).

Surge el conectivismo como un enfoque pedagógico para el desarrollo de procesos de aprendizaje que se pueden generar desde las tecnologías de la información y la comunicación aplicadas a la educación (Bernal-Garzón, 2020).

\section{LAS TECNOLOGÍAS DE LA INFORMACIÓN Y LA COMUNICACIÓN (TIC)}

Las llamadas Tecnologías de la Información y la Comunicación son los recursos y herramientas que se utilizan para el proceso, administración y distribución de la información a través de elementos tecnológicos, como: ordenadores, teléfonos, televisores, etc (Universidad Latina de Costa Rica, 2021).

Las tecnologías de la información y comunicación, son un conjunto de servicios en telecomunicación, coadyuvan el desarrollo sostenido y estratégico en cualquier ámbito rural o urbano. Sin embargo, requiere de plataformas complejas para su funcionamiento (Cusihuamán, Alarcón \& Ontiveros, 2020).

Las TIC han variado con el tiempo, debido a los rápidos avances que se suceden. Esto es consecuencia de los desarrollos tecnológicos en los máximos representantes de las TIC: los ordenadores, la informática, Internet y los smartphones. Su impacto social actual es innegable. Las características...: instantaneidad, interactividad, interconexión y diversidad, sin que por ello desaparezcan otras características que podemos considerar básicas o fundamentales (Grande, Cañón \& Cantón, 2016). 
Gracias a las nuevas tecnologías se emplean materiales y recursos de gran calidad por parte de los alumnos/as, a la vez que se consigue una participación muy activa del alumnado en la asignatura y en las tareas y actividades que se proponen en el aula, tanto a nivel individual como a nivel grupal (Rojano, López y López, 2016).

Las tecnologías de la información y la comunicación (TIC) pueden complementar, enriquecer y transformar la educación. En su calidad de agencia principal de las Naciones Unidas para la educación, la UNESCO orienta el quehacer internacional con miras a ayudar a los países a entender la función que puede desarrollar esta tecnología en acelerar el avance hacia el Objetivo de Desarrollo Sostenible 4(link is external) (ODS4), una visión plasmada en la Declaración de Qingdao (UNESCO, 2021).

La evolución de las TIC ha permitido que las actividades cotidianas sean más sencillas y rápidas en cada proceso de la vida real. Esta evolución tecnológica ha beneficiado a todos los seres humanos en las diferentes áreas en las cuales se desarrollan, como pueden ser el trabajo, educación, entretenimiento, negocios, entre otros (Guaña-Moya, Quinatoa-Arequipa \& Pérez-Fabara, 2017).

Las Tecnologías de la Información y la Comunicación (TIC) conllevan transformaciones y reestructuraciones que dan lugar a creación e intercambio del conocimiento así como nuevas formas de adquirir, abordar y organizar el proceso de formación (Cabero \& Llorente, 2015).

Las denominadas tecnologías digitales tienen significado siempre y cuando los individuos puedan hacer uso de las mismas de manera interconectada, es decir cuando accede a internet y con ello aprovechar dicho recurso en función de su realidad y requerimientos; por ello se deben de generar una serie de políticas y medidas económicas que tiendan a revertir el proceso de estática respecto al acceso a internet y en especial del equipamiento, en relación a la mejora o renovación de los mismos en la población (Flores-Cueto, Hernández y Garay-Argandoña, 2020).

Los resultados apuntan a que las TIC han aportado considerablemente a diferentes aspectos neurálgicos, en el contexto educativo y que las aplicaciones en los procesos pedagógicos son cada vez mayores (De-la-Hoz-Franco, Martínez-Palmera, Combita-Niño \& Hernández-Palma, 2019).

El periodo de confinamiento ha provocado cambios notables en el estilo de vida de los niños y sus hábitos sociales. La presencia de un mayor o menor número de TIC en el hogar y su uso puede influir en otras actividades realizadas por los niños (Arufe, Cachón, Zagalaz, Sanmiguel-Rodríguez \& González, 2020).

Los beneficios que traen las TIC en la educación, han dejado al descubierto la imperiosa necesidad de capacitación y actualización docente, para enfrentar con exigencia las nuevas circunstancias que ha traído el Covid19 a los procesos de formación universitarios. El evidente protagonismo de la tecnología en la educación, genera cambios significativos tanto en las estrategias utilizadas para la 
enseñanza, como en el modelo comunicacional para mantener la calidad del servicio educativo en las actuales circunstancias (Poveda-Pineda \& Cifuentes-Medina, 2020).

Las Tecnologías de la Información y la Comunicación son utilizadas intensamente en las organizaciones pública y privadas a nivel mundial, mostrando su valía durante la pandemia vigente. Han permitido que las instituciones educativas puedan continuar interactuando con los estudiantes.

\section{EL CONECTIVISMO}

El conectivismo plantea que el conocimiento se encuentra distribuido a través de una red personal de conexiones, y por consiguiente el aprendizaje consiste en la habilidad de los individuos para construir y navegar esas redes (Sánchez, 2019).

El conectivismo tiene como idea central que el conocimiento se distribuye a través de una red de conexiones y, por lo tanto, el aprendizaje consiste en la capacidad de construir y atravesar esas redes. La inclusión de la tecnología y la identificación de conexiones como actividades de aprendizaje empiezan a mover a las teorías de aprendizaje hacia la era digital. Ya no es posible experimentar y adquirir personalmente el aprendizaje que necesitamos para actuar (Solórzano \& García, 2016).

El conectivismo se orienta como una teoría de aprendizaje de la nueva era digital, aborda estrategias de aprendizaje que cubran las expectativas actuales de los estudiantes como herramienta innovadora en el proceso enseñanza-aprendizaje del idioma Inglés, conlleva a la búsqueda de una pedagogía y de la elaboración de actividades que alienten a los estudiantes a desarrollar habilidades como nativos digitales (Basurto, Moreira, Velásquez y Rodríguez, 2021).

Desde mi punto de vista, observo que el conectivismo también ayuda a las personas a entender que el aprendizaje no es algo basado en texto, en lógica y lenguaje. El aprendizaje abarca todos los sentidos. Algunos dirían que aprender no es solo algo que se conoce, sino que se incorpora1: el aprendizaje es un proceso de crecimiento y desarrollo, en lugar de adquisición y creación. Creo que esas son las cuestiones principales que señalaría en este momento (Vadillo, 2018).

El conectivismo presenta un modelo de aprendizaje que reconoce los movimientos tectónicos en una sociedad en donde el aprendizaje ha dejado de ser una actividad interna e individual. La forma en la cual trabajan y funcionan las personas se altera cuando se usan nuevas herramientas. El área de la educación ha sido lenta para reconocer el impacto de nuevas herramientas de aprendizaje y los cambios ambientales, en la concepción misma de lo que significa aprender. El conectivismo provee una mirada a las habilidades de aprendizaje y las tareas necesarias para que los aprendices florezcan en una era digital (Siemens, 2004).

Partiendo de esta premisa, aprender en la era digital implica ser parte de redes que se conectan bajo sentidos comunes, con nodos que comparten contenidos y conocimientos de acuerdo con un 
contexto o ámbito de interés, que cubren las necesidades de aprender de un individuo...También, este proceso se realiza apoyado por las tecnologías de la información que adquieren el papel de depositarias de la información y conocimiento, así como de transportadoras, receptoras y expositoras (Islas, 2021).

Según Siemens (2004) los principios del conectivismo son:

- El aprendizaje y el conocimiento dependen de la diversidad de opiniones.

- El aprendizaje es un proceso de conectar nodos o fuentes de información especializados.

- El aprendizaje puede residir en dispositivos no humanos.

- La capacidad de saber más es más crítica que aquello que se sabe en un momento dado.

- La alimentación y mantenimiento de las conexiones es necesaria para facilitar el aprendizaje continuo.

- La habilidad de ver conexiones entre áreas, ideas y conceptos es una habilidad clave.

- La actualización (conocimiento preciso y actual) es la intención de todas las actividades conectivistas de aprendizaje.

- La toma de decisiones es, en sí misma, un proceso de aprendizaje. El acto de escoger qué aprender y el significado de la información que se recibe, es visto a través del lente de una realidad cambiante. Una decisión correcta hoy, puede estar equivocada mañana debido a alteraciones en el entorno informativo que afecta la decisión.

Las ventajas del conectivismo son: Continuidad respecto al conocimiento educativo existente..., facilidad de aplicación de los principios conectivistas a las nuevas tecnologías y metodologías educativas..., alta capacidad de adaptación a las nuevas generaciones de alumnos 'Nativos Digitales'..., mayor sintonía entre las distintas ciencias y campos de estudios..., mayor comprensión de la situación educativa actual y los desafíos futuros...(Sánchez-Cabrero, Costa-Román, Mañoso-Pacheco, Novillo-López \& Pericacho-Gómez).

En la figura 1 se muestra el mapa conceptual del conectivismo, que ayuda a comprender su importancia en la era digital. 


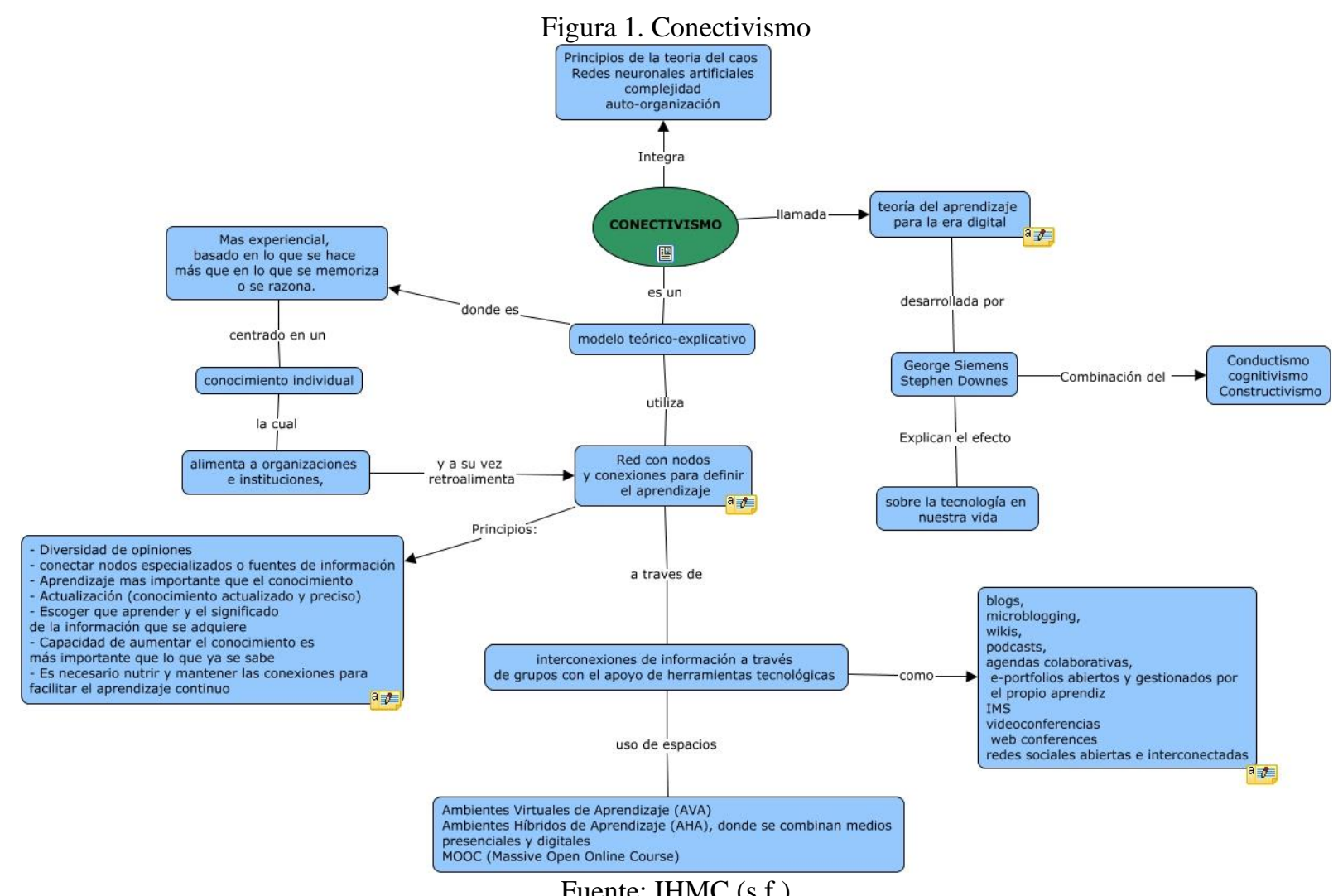

A su vez, el proceso de adquisición de las competencias informacionales, las habilidades de interpretación visual y la comprensión de las ideas del conectivismo, requieren de un periodo prolongado que, al finalizarse, permite al/la estudiante adecuarse a los cambios tecnológicos y educacionales que surgieran (Oliveros-Castro y Núñez-Chaufleur, 2020).

Se evidencian pedagogías emergentes enfocadas en la incidencia del conectivismo en las ecologías del aprendizaje, los ecosistemas, el actor-red, el eco-conectivismo y, el aprendizaje nómada. Entre otras pedagogías que se contextualizan en comunidades de práctica y los ecosistemas educativos (Ortiz y Corrêa, 2020).

En la figura 2 se muestra el modelo elaborado por Cueva, García \& Martínez (2019) que permite comprender la interrelación entre las Tecnologías de la Información y la Comunicación y el conectivismo. Este modelo resulta muy importante en el proceso de enseñanza aprendizaje actual. 
Figura 2. Modelo de la influencia del conectivismo para el uso de las TIC en el proceso de enseñanza aprendizaje.

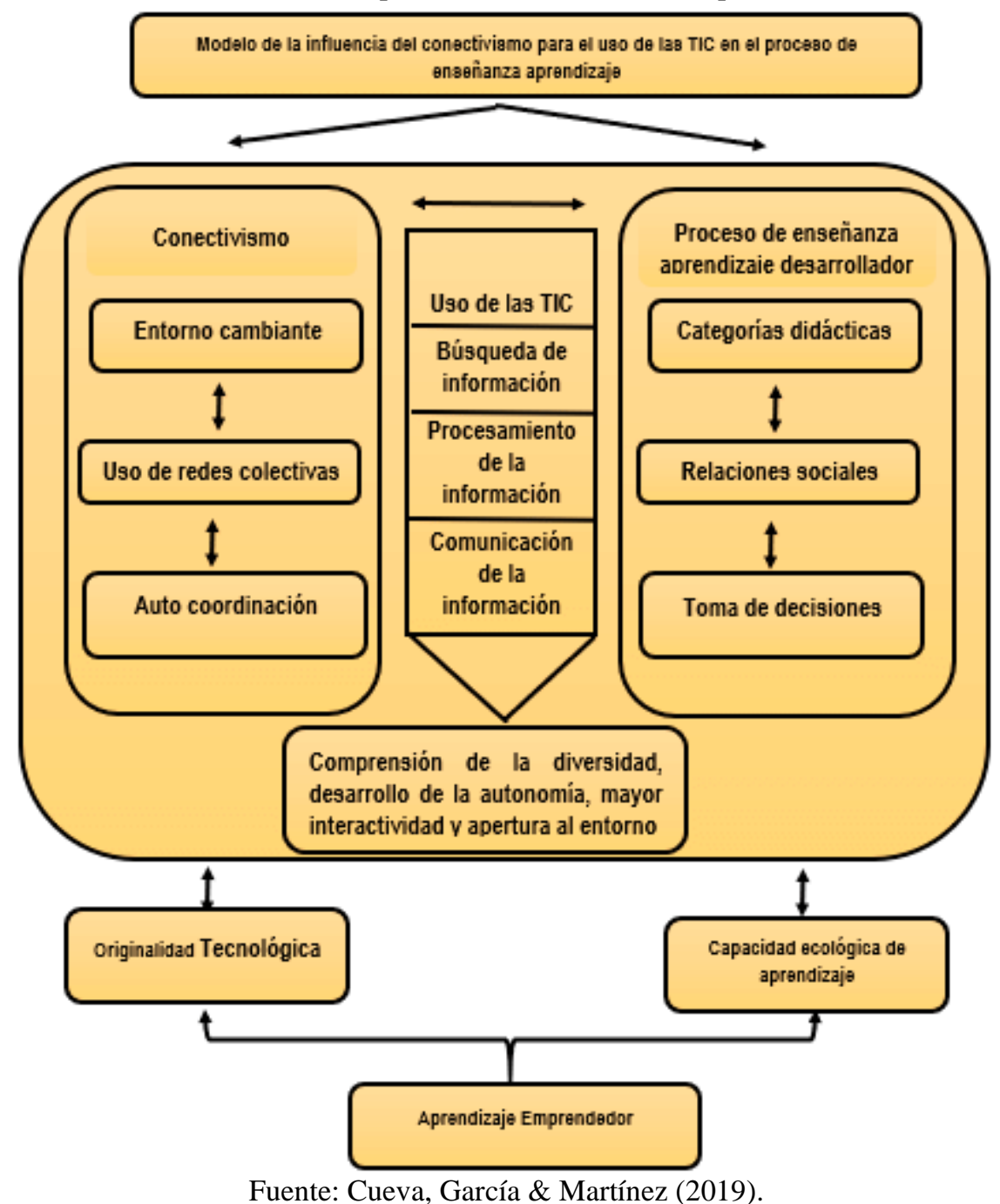

Fuente: Cueva, García \& Martínez (2019).

\section{METODOLOGÍA}

La investigación presenta un diseño cualitativo-interpretativo, de tipo documental, el cual precisó el procedimiento de selección y el registro de los datos (Barrero y Rosero, 2018).

En la presente investigación se han seleccionado 47 documentos, realizados en el periodo 2016 2021; incluyendo: artículos científicos, artículos de revisión e información de sitios web de organizaciones reconocidas; a excepción de un documento del año 2005, de un autor referente en el tema. Las palabras claves utilizadas en las búsquedas fueron: Tecnologías de la Información y la Comunicación, conectivismo y educación. Para la selección de los documentos se usaron como criterios: el año de la publicación, la pertenencia con la investigación y ser una fuente confiable. Después de la lectura de cada documento, se procedió a ingresar los datos a la matriz bibliográfica, que sirve para catalogar los documentos de acuerdo con categorías, que se presentan en la figura 3. 
Figura 3. Matriz bibliográfica

\begin{tabular}{|l|l|l|l|}
\hline Nombre & Tipo & Objetivos & Conclusiones \\
\hline & & & \\
\hline & & & \\
\hline
\end{tabular}

Fuente: Adaptado de Barrero y Rosero (2018).

\section{RESULTADOS}

El impacto y las implicaciones de la revolución digital se hacen cada vez más evidentes y la Agenda 2030 para el Desarrollo Sostenible reconoce el gran potencial de la conectividad global para estimular el desarrollo humano (Naciones Unidas, 2017).

Las tecnologías de la información y la comunicación (TIC) pueden contribuir a acelerar el cumplimiento de cada uno de los 17 Objetivos de Desarrollo Sostenible de las Naciones Unidas (ODS).

La UIT, en colaboración con sus asociados, ha estado trabajando para contribuir a todos y cada uno de los ODS: ODS 1: Erradición de la pobreza, ODS 2: Hambre cero, ODS 3: Buena saludo y bienestar, ODS 4: Educación de calidad, ODS 5: Igualdad de género, ODS 6: Agua limpia y saneamiento, ODS 7: Energía limpia y asequible, ODS 8: Trabajo digno y crecimiento económico, ODS 9: Industria, innovación e infraestructura, ODS 10: Reducción de las desigualdades, ODS 11: Ciudades y comunidades sostenibles, ODS 12: Consumo y producción, ODS 13: Acción contra el cambio climático, ODS 13, 14 y 15 - Acción climática, vida marina y vida Terrestre, ODS 16: Paz, justicia e instituciones sólidas, ODS 17: El poder de las asociaciones (UIT, 2018).

Figura 4. Objetivos de Desarrollo Sostenible (ODS)
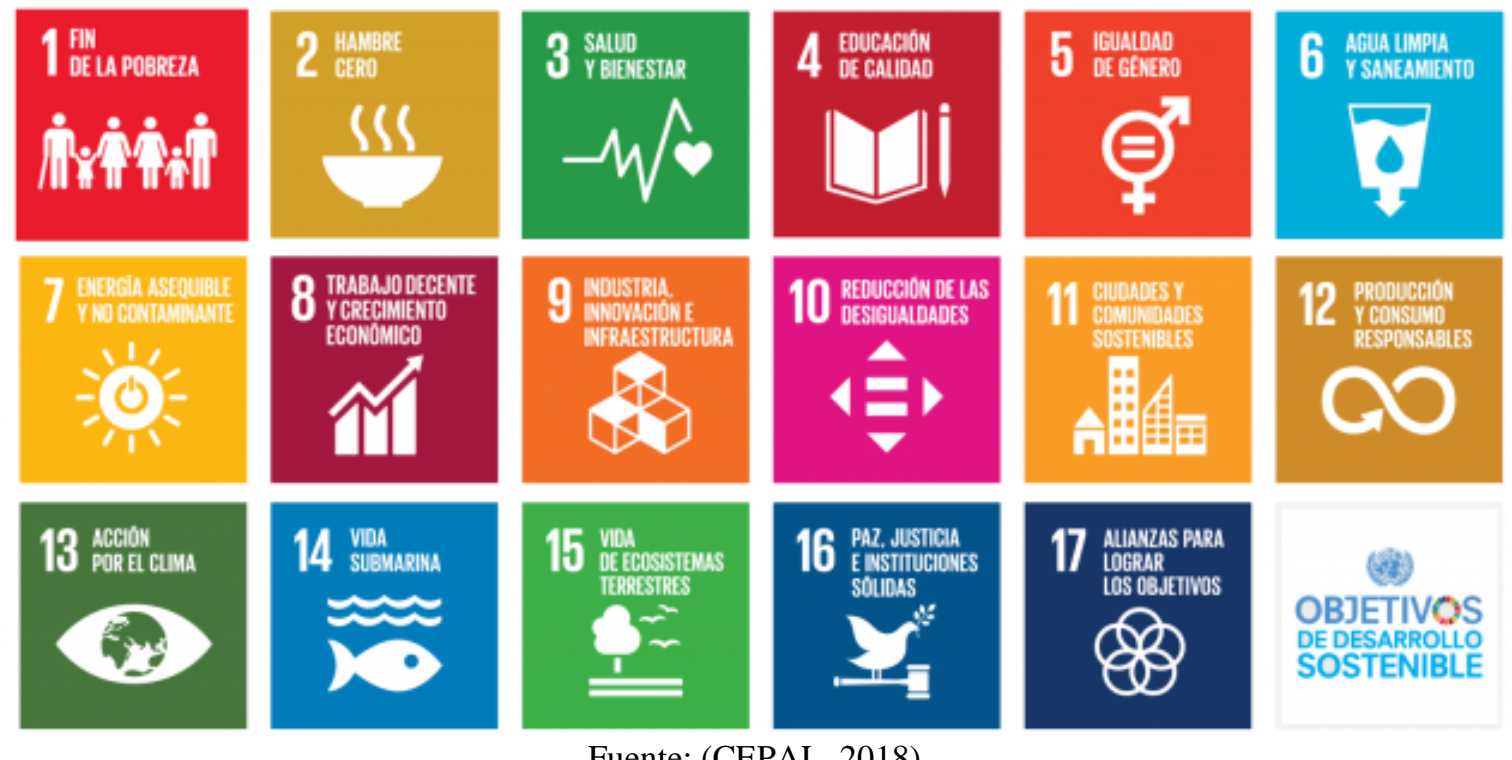

OBJETIVOS

DEDESARROLLO

Fuente: (CEPAL, 2018)

SOSTENIBLE 
Tabla 1. Disponibilidad de Información sobre TIC en los censos de población y vivienda para la década 2010

\begin{tabular}{|c|c|c|c|c|c|c|c|}
\hline PAIS & DECADA & Radio & TV & Tel fijo & Móvil & PC & Internet \\
\hline Argentina & 2010 & & & $\mathrm{Si}$ & Si & Si & \\
\hline Bolivia (Est.Plur.de) & 2010 & $\mathrm{Si}$ & $\mathrm{Si}$ & $\mathrm{Si}$ & Si & $\mathrm{Si}$ & $\mathrm{Si}$ \\
\hline Brasil & 2010 & Si & Si & $\mathrm{Si}$ & Si & Si & Si \\
\hline Chile & 2010 & & & & & & \\
\hline Costa Rica & 2010 & $\mathrm{Si}$ & $\mathrm{Si}$ & $\mathrm{Si}$ & $\mathrm{Si}$ & $\mathrm{Si}$ & $\mathrm{Si}$ \\
\hline Cuba & 2010 & Si & Si & $\mathrm{Si}$ & Si & $\mathrm{Si}$ & \\
\hline Ecuador & 2010 & & $\mathrm{Si}$ & $\mathrm{Si}$ & $\mathrm{Si}$ & $\mathrm{Si}$ & $\mathrm{Si}$ \\
\hline Honduras & 2010 & $\mathrm{Si}$ & $\mathrm{Si}$ & $\mathrm{Si}$ & $\mathrm{Si}$ & $\mathrm{Si}$ & Si \\
\hline México & 2010 & Si & $\mathrm{Si}$ & Si & Si & Si & Si \\
\hline Panamá & 2010 & $\mathrm{Si}$ & $\mathrm{Si}$ & $\mathrm{Si}$ & $\mathrm{Si}$ & $\mathrm{Si}$ & $\mathrm{Si}$ \\
\hline Paraguay & 2010 & Si & Si & $\mathrm{Si}$ & Si & $\mathrm{Si}$ & $\mathrm{Si}$ \\
\hline Perú & 2010 & $\mathrm{Si}$ & $\mathrm{Si}$ & $\mathrm{Si}$ & Si & Si & Si \\
\hline República Dominicana & 2010 & $\mathrm{Si}$ & Si & $\mathrm{Si}$ & Si & $\mathrm{Si}$ & $\mathrm{Si}$ \\
\hline Uruguay & 2010 & $\mathrm{Si}$ & Si & $\mathrm{Si}$ & Si & $\mathrm{Si}$ & $\mathrm{Si}$ \\
\hline Venezuela (Rep.Bol.de) & 2010 & $\mathrm{Si}$ & $\mathrm{Si}$ & $\mathrm{Si}$ & $\mathrm{Si}$ & Si & $\mathrm{Si}$ \\
\hline
\end{tabular}

Fuente: Silva (2018)

Determinar que las TIC no sólo entran a jugar un papel importante en el proceso educativo, sino examinar que realmente están presentes y se requieren en los procesos de gestión, facilitando de cierta forma el manejo eficiente y eficaz de la información es tema analizado en la parte administrativoacadémica (Nájar, 2016).

Un caso interesante en Perú, es la Biblioteca Central Jaime Hernández de Souza de la Universidad Nacional "Pedro Ruiz Gallo" que requiere mayor presupuesto anual para la capacitación de los trabajadores, inversión en infraestructura y equipamiento tecnológico; los trabajadores no tienen acceso a información multimedia; debe utilizar las TIC's; se consideró a la Teoría General de Sistemas y un Marco Conceptual; se determinó los componentes: Sociedad, universidad, información multimedia, TIC's, recursos humanos, económicos y materiales. Y las relaciones: Sociedad - Biblioteca Central, Universidad - Biblioteca Central y Relaciones Internas (Ríos, 2017).

El docente cumple un rol fundamental en el conocimiento y uso de estas tecnologías, debido a la importancia didáctica que pueden tener las TIC empleadas adecuadamente en el aula de clase. Sin embargo, los docentes hoy en día no comprenden con eficacia la utilidad que puede significar el uso de las TIC en el desarrollo del proceso de formación de los estudiantes; esto se refleja en la calidad educativa (Cruz Pérez, Pozo Vinueza, Aushay Yupangui, y Arias Parra, 2019).

Los resultados indican que su aprendizaje se ve influenciado por las características del contexto en que se desarrollan; están rodeados de tecnología, información, redes de comunicación, etcétera; por tanto, la construcción del conocimiento se da en términos de lo que los estudiantes logran compartir, 
colaborar, discutir o reflexionar con sus compañeros y docentes sobre temas de su interés, aunque la retroalimentación no es tan alta como se esperaría (Islas y Delgadillo, 2016).

Los resultados mostraron que, a pesar de ser profesores universitarios, el conocimiento que poseen respecto a las tecnologías de la información y las comunicaciones es bajo, a pesar de todas las posibilidades que se brindan en el proceso docente educativo, por lo que se corresponde con el insuficiente uso que realizan de las mismas, aunque la actitud fue positiva (Almanza, Soler, Mesa, Naranjo \& Soler, 2021).

El instrumento fue aplicado a estudiantes de dos centros de educación superior ubicados en México y Costa Rica. Los principales resultados señalan que el estudiantado valora las TIC como herramientas significativas en su formación; sin embargo, se excluye a estudiantes que no tienen un fuerte desempeño en estas tecnologías (Patricia, Chévez-Ponce \& Oviedo-Vargas, 2020).

El uso de esta TIC potenció las capacidades de esos estudiantes, constatando mejoras cognitivas para el aprendizaje de los tópicos de lógico matemática, comunicación y ciencias sociales. Asimismo, se constató que esta herramienta permite un mejor y mayor desarrollo de sus habilidades sociales (VértizOsores, Pérez-Saavedra, Faustino-Sánchez, Vértiz-Osores, \& Alain, 2019).

Las TIC, aplicadas en clase y el trabajo independiente, inciden positivamente en la calidad del proceso de aprendizaje. Los resultados de docentes, estudiantes y directivos fueron confrontados llegándose a determinar con esta triangulación, que debe realizarse un programa de capacitación, determinándose los temas que deben ser considerados en un programa de capacitación para los docents (ílvarez, Pesantes \& Salazar, 2017).

Exponer los factores que inciden en el uso de las tecnologías de la información y la comunicación (TIC) por parte de los docentes de Educación Básica, lo que se ha convertido en un dilema profesional, debido a causas externas como la baja disponibilidad o ausencia de centros, software, equipos tecnológicos e Internet (Martínez, 2018).

Se deben diseñar estrategias adecuadas que permitan a las Instituciones de Educación Superior, tener la certeza de que los estudiantes las han adquirido. Para esto, se debería determinar los niveles de competencias digitales que poseen los estudiantes cuando llegan a la universidad y a partir de esta línea de base, poder garantizar, a través de su formación, el desarrollo de las mismas hasta finalizar sus estudios regulares (Humanante-Ramos, Solís-Mazón, Fernández-Acevedo y Silva-Castillo, 2019).

Algunos de los resultados encontrados muestran que sí existen diferencias significativas en cuestiones de género, mostrando las mujeres mayores habilidades en movilización de herramientas y los hombres en habilidades técnicas-tecnológicas, así como diferencias con respecto a la edad, entre otras (Cabero, Barroso, Llorente \& Yanes, 2016). 
La investigación demuestra que el valor pedagógico en el uso de las TIC es un factor clave para la introducción de la enseñanza remota y un avance en los procesos de aprendizaje mediados por las TIC (Avendaño, Hernández \& Prada, 2021).

Asu vez, se constata un relevante y gradual cambio en los últimos años en cuanto al lugar de conexión a internet, desde el hogar como el principal lugar de conexión al telefono móvil como medio más frecuente Por último, como otro hallazgo significativo, es que en los hombres se refleja una disminución en el tiempo dedicado a realizar tareas académicas y un aumento en el tiempo dedicado a jugar (Ordóñez, Vázquez-Cano, Arias-Sánchez \& López-Meneses, 2021).

La incorporación de TIC, como herramientas educativas para los estudiantes universitarios, muestra ser relevante en su proceso de formación. Con la utilización de éstas, adquieren habilidades y actitudes para enfrentarse a su futuro laboral, contribuyendo al mejoramiento de la calidad de la educación (Faúndez, Bravo, Ramírez \& Astudillo, 2017).

Se propone un modelo de la influencia del conectivismo para el uso de las TIC en el proceso de enseñanza aprendizaje, en el que se establecen nuevas relaciones entre los elementos que componen el conectivismo y las categorías del proceso de enseñanza aprendizaje desarrollador, en el marco del buen vivir, así como, que se describen los nuevos rasgos que caracterizan al modelo y se define como cualidad resultante entre las relaciones de los componentes del modelo pedagógico de Aprendizaje Emprendedor (Cueva, García \& Martínez, 2019).

El conectivismo como una teoría de aprendizaje de la nueva era digital, ayuda a mejorar el uso de las TIC, recordando que estas resultan herramientas muy importantes para los estudiantes de los diferentes niveles educativos.

\section{CONCLUSIONES}

Las TICS han permitido un cambio sustancial en el sector educativo como un ente facilitador del conocimiento con metodologías participativas, uso de plataformas educativas integrando estrategias tecnológicas innovadoras, que han logrado impactar en la formación de calidad del estudiante, cambiando su comportamiento cultural y social, logros en la investigación rápida y oportuna al fortalecer su aprendizaje, habilidades de búsqueda, canales de comunicación, interactividad y ahorro de tiempo (Núñez, 2021).

El proceso de enseñanza-aprendizaje en el aula, haciendo uso de las TIC, requiere de un conjunto de competencias que el docente debe adquirir con la lógica de sumar una metodología capaz de aprovechar las herramientas tecnológicas, donde la capacitación docente deberá considerarse una de las primeras opciones antes de afrontar nuevos retos educativos (Hernandez, 2017). 
De manera que el conectivismo tiene mucho camino por andar, por ejemplo, la vía de la Didáctica crítica, por donde la auto-organización, la complejidad y el caos, propios de esa teoría, logren ser orientados en función de una verdadera utilidad para los procesos de enseñanza-aprendizaje. Para ello, sería fundamental la implantación de «aulas virtuales creativas», de cuya creación se tiene conocimiento, pero sobre las cuales su impacto ha sido escasamente evaluado o validado en función del PEA (Pando, 2018).

Las Tecnologías de la Información y la Comunicación y el conectivismo, son muy importantes en esta era digital, donde los nativos digitales tienen muchas ventajas en comparación a los migrantes digitales. En nuestros países latinoamericanos, la brecha digital limita la adopción de las TIC por las grandes mayorías. 


\section{REFERENCIAS}

Almanza Santana, Lorayne, Soler Cárdenas, Silvio, Mesa Simpson, Cristóbal, Naranjo Rodríguez, Sandra, \& Soler Pons, Lisbet. (2021). El uso de las nuevas tecnologías de la información y las comunicaciones por los profesores de las ciencias médicas en Matanzas. Revista Médica Electrónica, 43(1), 2917-2927. Epub 28 de febrero de 2021. Recuperado en 20 de septiembre de 2021, de http://scielo.sld.cu/scielo.php?script=sci_arttext\&pid=S1684-18242021000102917\&lng=es\&tlng=es.

Arufe Giráldez, V., Cachón Zagalaz, J., Zagalaz Sánchez, M. L., Sanmiguel-Rodríguez, A., \& González Valero, G. (2020). Equipamiento y uso de Tecnologías de la Información y Comunicación (TIC) en los hogares españoles durante el periodo de confinamiento. Asociación con los hábitos sociales, estilo de vida y actividad física de los niños menores de 12 años. Revista Latina De Comunicación Social, (78), 183-204. https://doi.org/10.4185/RLCS-2020-1474

Avendaño Castro, W. R., Hernández S., C. A., \& Prada Núñez, R. (2021). Uso de las Tecnología de Información y Comunicación como valor pedagógico en tiempos de crisis. Revista Historia De La Educación Latinoamericana, 23(36). https://doi.org/10.19053/01227238.11619

Basurto, Shirley, Moreira, José, Velásquez, Angélica y Rodríguez, María (2021). El conectivismo como teoría innovadora en el proceso de enseñanza-aprendizaje del idioma inglés. Pol. Con. (Edición núm. 54) Vol. 6, No 1. Enero 2021, pp. 234-252. DOI: 10.23857/pc.v6i1.2134

Bernal-Garzón, E. (2020). Aportes a la consolidación del conectivismo como enfoque pedagógico para el desarrollo de procesos de aprendizaje. Revista Innova Educación, 2(3), 394-412. https://doi.org/10.35622/j.rie.2020.03.002

Cabero Almenara, Julio, \& Llorente Cejudo, María del Carmen. (2015). Tecnologías de la Información y la Comunicación (TIC): escenarios formativos y teorías del aprendizaje. Revista Lasallista de Investigación, 12(2), 186-193. Recuperado de http://www.scielo.org.co/scielo.php?script=sci_arttext\&pid=S179444492015000200019\&lng=en\&tlng=es.

Cabero Almenara, J., Barroso Osuna, J., Llorente Cejudo, M. del C., \& Yanes Cabrera, C. (2016). Redes sociales y Tecnologías de la Información y la Comunicación en Educación: aprendizaje colaborativo, diferencias de género, edad y preferencias. Revista de Educación a Distancia (RED), (51). Recuperado a partir de https://revistas.um.es/red/article/view/275131

Cabezas, César. (2020). Pandemia de la COVID-19: tormentas y retos. Revista Peruana de Medicina Experimental y Salud Pública [online]. 2020, v. 37, n. 4, pp. 603-604. Recuperado de https://doi.org/10.17843/rpmesp.2020.374.6866

CEPAL (2018). La Agenda 2030 y los Objetivos de Desarrollo Sostenible Una oportunidad para América Latina $\quad y \quad$ el Caribe. $\quad$ Recuperado de https://repositorio.cepal.org/bitstream/handle/11362/40155/24/S1801141_es.pdf

Córdova-Aguilar, Alberto, \& Rossani A., Germán. (2020). COVID-19: Revisión de la literatura y su impacto en la realidad sanitaria peruana. Revista de la Facultad de Medicina Humana, 20(3), 471-477. https://dx.doi.org/10.25176/rfmh.v20i3.2984

Cruz Pérez, M.A., Pozo Vinueza, M.A., Aushay Yupangui, H.R. y Arias Parra, A.D. (2019). Las Tecnologías de la Información y de la Comunicación (TIC) como forma investigativa interdisciplinaria con un enfoque intercultural para el proceso de formación estudiantil. e-Ciencias de la Información, 9(1). doi: https://doi.org/10.15517/eci.v1i1.33052 
Cueva Delgado, J. L., García Chávez, A., \& Martínez Molina, O. A. (2019). El conectivismo y las TIC: Un paradigma que impacta el proceso enseñanza aprendizaje. Revista Scientific, 4(14), 205-227. https://doi.org/10.29394/Scientific.issn.2542-2987.2019.4.14.10.205-227

Cusihuamán SisaG. N., Alarcón CondoriJ. G., \& Ontiveros AparicioW. R. (2020). Tecnologías de la información y comunicación, interculturalidad y desarrollo rural en la provincia de la unión, Arequipa Perú. PUBLICACIONES, 50(2), 15-29. https://doi.org/10.30827/publicaciones.v50i2.13940

De-la-Hoz-Franco, Emiro, Martínez-Palmera, Olga, Combita-Niño, Harold, \& Hernández-Palma, Hugo. (2019). Las Tecnologías de la Información y la Comunicación y su Influencia en la Transformación de la Educación Superior en Colombia para Impulso de la Economía Global. Información tecnológica, 30(1), 255262. https://dx.doi.org/10.4067/S0718-07642019000100255

Faúndez, Claudio A, Bravo, Alicia A, Ramírez, Glenda P, \& Astudillo, Hernán F. (2017). Tecnologías de la Información y la Comunicación (TIC) en el Proceso de Enseñanza-Aprendizaje de Conceptos de Termodinámica como Herramienta para Futuros Docentes. Formación universitaria, 10(4), 43-54. https://dx.doi.org/10.4067/S0718-50062017000400005

Flores-Cueto, J., Hernández, R. y Garay-Argandoña, R. (2020). Tecnologías de información: Acceso a internet y brecha digital en Perú. Revista Venezolana de Gerencia, vol. 25, núm. 90, pp. 504-527. Recuperado de https://www.redalyc.org/journal/290/29063559007/html/

Grande, M., Cañón, R., \& Cantón, I. (2016). Tecnologías de la información y la comunicación: Evolución del concepto y características. IJERI: International Journal of Educational Research and Innovation, (6), 218230. Recuperado de https://www.upo.es/revistas/index.php/IJERI/article/view/1703

Guaña-Moya, Edison Javier, \& Quinatoa-Arequipa, Edwin, \& Pérez-Fabara, María Augusta (2017). Tendencias del uso de las tecnologías y conducta del consumidor tecnológico. Ciencias Holguín, 23(2),1530. Disponible en: https://www.redalyc.org/articulo.oa?id=181550959002

Hernandez, R. M. (2017). Impacto de las TIC en la educación: Retos y Perspectivas. Propósitos y Representaciones, 5(1), 325 - 347 http://dx.doi.org/10.20511/pyr2017.v5n1.149

Humanante-Ramos, P., Solís-Mazón, M., Fernández-Acevedo, J. y Silva-Castillo, J. (2019). Las competencias TIC de los estudiantes que ingresan en la universidad: una experiencia en la Facultad de Ciencias de la Salud de una universidad latinoamericana, Educación Médica, Volume 20, Issue 3. Pages 134139. https://doi.org/10.1016/j.edumed.2018.02.002.

IHMC (s.f.). Conectivismo. Recuperado de https://skat.ihmc.us/rid=1PW5QDKZL-1GTLGWB2N8T/Conectivismo.cmap

Ílvarez Betancourt, J. M., Pesantes Piguave, M. E., \& Salazar Quinatoa, K. J. (2017). Incidencia de las tecnologías de la información y comunicación (TIC) en la calidad del proceso de aprendizaje presencial.

Revista Publicando, 4(13 (2), 253-268. Recuperado de https://revistapublicando.org/revista/index.php/crv/article/view/844

Islas, C. (2021). Conectivismo y neuroeducación: transdisciplinas para la formación en la era digital. CIENCIA ergo-sum, Revista Científica Multidisciplinaria de Prospectiva, vol. 28, núm. 1. https://doi.org/10.30878/ces.v28n1a11 
Islas, C. y Delgadillo, O. (2016). La inclusión de TIC por estudiantes universitarios: una mirada desde el conectivismo. Apertura Vol. 8, Núm. $2 . \quad$ Recuperado de http://www.udgvirtual.udg.mx/apertura/index.php/apertura/article/view/845/581

Macias Llanes, María Elena. (2020). COVID- 19: La respuesta social a la pandemia. Humanidades Médicas, 20(1), 1-4. Epub 29 de marzo de 2020. Recuperado en 18 de septiembre de 2021, de http://scielo.sld.cu/scielo.php?script=sci_arttext\&pid=S1727-81202020000100001\&lng=es\&tlng=es.

Martínez Molina, O. A. (2018). Uso de las Tecnologías de la Información y la Comunicación en la Educación Básica. Revista Scientific, 3(10), 154-174. https://doi.org/10.29394/Scientific.issn.25422987.2018.3.10.8.154-174

Naciones Unidas (2017). Las tecnologías de la información son fundamentales para responder a los desafíos del desarrollo. Recuperado de https://www.un.org/sustainabledevelopment/es/2017/07/las-tecnologias-de-lainformacion-son-fundamentales-para-responder-a-los-desafios-del-desarrollo/

Nájar, O. (2016). Tecnologías de la información y la comunicación aplicadas a la educación. Praxis \& Saber, vol. 7, núm. 14, pp. 9-16, 2016. Universidad Pedagógica y Tecnológica de Colombia. DOI: https://doi.org/http://dx.doi.org/10.19053/22160159.5215

Núñez Cheng, J. (2021). Tecnologías de la Información y Comunicación en el Desarrollo de las Competencias Matemáticas en la Educación Virtual Universitaria. Ciencia Latina Revista Científica Multidisciplinar, 5(3), 2908-2930. https://doi.org/10.37811/cl_rcm.v5i3.497

Oliveros-Castro, S. y Núñez-Chaufleur, C. (2020). Posibilidades educativas de la realidad virtual y la realidad combinada: una mirada desde el conectivismo y la bibliotecología. Revista Saberes Educativos № 5, JULIODICIEMBRE 2020, PP. 46-62. https://revistas.uchile.cl/index.php/RSED/article/download/57783/63384/

Ordóñez, E., Vázquez-Cano, E., Arias-Sánchez, S. \& López-Meneses, E. (2021). Las Competencias en el uso de las Tecnologías de la Información y la Comunicación en el alumnado universitario. Píxel-Bit. Revista de Medios y Educación, 60, 153-167. https://doi.org/10.12795/pixelbit.74860

Ortiz, J. y Corrêa, T. (2020). Aspectos pedagógicos del conectivismo y su relación con redes sociales y ecologías del aprendizaje. Revista Brasileira de Educação v. 25. https://doi.org/10.1590/S141324782020250026

Pando, V. (2018). Tendencias didácticas de la educación virtual: Un enfoque interpretativo. Propósitos y Representaciones, 6(1), 463-505. https://dx.doi.org/10.20511/pyr2018.v6n1.167

Patricia Astudillo-Torres, M., Chévez-Ponce, F., \& Oviedo-Vargas, Y. (2020). La Exclusión Social Y Las Tecnologías De La Información Y La Comunicación: Una Visión Estadística De Su Relación en La Educación Superior. Liminar, 18(1), 177-193. https://doi.org/10.29043/liminar.v18i1.721

Poveda-Pineda, Derly F., \& Cifuentes-Medina, José E. (2020). Incorporación de las tecnologías de información y comunicación (TIC) durante el proceso de aprendizaje en la educación superior. Formación universitaria, 13(6), 95-104. https://dx.doi.org/10.4067/S0718-50062020000600095

Ríos, C. (2017). Modelo de gestión de las TIC’s de la Biblioteca Central Jaime Hernández de Souza de la Universidad Nacional "Pedro Ruiz Gallo". UCV - HACER: Revista de Investigación y Cultura, Vol. 6, No. 1, pp. 40-47. Recuperado de https://dialnet.unirioja.es/servlet/articulo?codigo=6090854 
Rojano, S., López, M. y López, G. (2016). Desarrollo de tecnologías de la información y la comunicación para reforzar los procesos de enseñanza y aprendizaje en ciencias en el grado de maestro/a en educación infantil de la Universidad de Málaga, Educación Química. Volume 27, Issue 3. Pages 226-232. https://doi.org/10.1016/j.eq.2016.04.006.

Sánchez-Cabrero, R., Costa-Román, O., Mañoso-Pacheco, L., Novillo-López, M \& Pericacho-Gómez, F. (2019). Orígenes del conectivismo como nuevo paradigma del aprendizaje en la era digital. Educación y Humanismo, 21(36), 113-136.DOI: http://dx10.17081/eduhum.21.36.3265

Sánchez Sordo, J. M. (2019). Desarrollo de un entorno digital de aprendizaje desde el Conectivismo y su posterior análisis utilizando algoritmos de machine learning. Edutec. Revista Electrónica de Tecnología Educativa, (69), 1-22. https://doi.org/10.21556/edutec.2019.69.1355

Siemens, G. (2004). Conectivismo: Una teoría de aprendizaje para la era digital. Recuperado de https://www.comenius.cl/recursos/virtual/minsal_v2/Modulo_1/Recursos/Lectura/conectivismo_Siemens.pd $\mathrm{f}$

Silva, A. (2018). Una mirada regional al acceso y tenencia de tecnologías de la información y comunicaciones - TIC, a partir de los censos. Recuperado de https://www.cepal.org/es/enfoques/mirada-regional-al-accesotenencia-tecnologias-la-informacion-comunicaciones-tic-partir

Solórzano, F., \& García, A. (2016). Fundamentos del aprendizaje en red desde el conectivismo y la teoría de la actividad. Revista Cubana de Educación Superior, 35(3), 98-112. Recuperado de http://scielo.sld.cu/scielo.php?script=sci_arttext\&pid=S0257-43142016000300008\&lng=es\&tlng=es.

UIT (2018). Las TIC para el cumplimiento de los Objetivos de Desarrollo Sostenible de las Naciones Unidas. Recuperado de https://www.itu.int/es/mediacentre/backgrounders/Pages/icts-to-achieve-the-united-nationssustainable-development-goals.aspx

UNESCO (2021). Las TIC en la educación. Recuperado de https://es.unesco.org/themes/tic-educacion Universidad Latina de Costa Rica (2021). Qué son las TIC y para qué sirven. Recuperado de https://www.ulatina.ac.cr/blog/qu3-son-las-tic-y-para-que-sirven

Vadillo, G. (2018). Stephen Downes y el conectivismo. Revista Mexicana de Bachillerato a Distancia, número 19, año 10, febrero de 2018. http://dx.doi.org/10.22201/cuaed.20074751e.2018.19.64909

Valverde, M. T. (2018). Escritura académica con Tecnologías de la Información y la Comunicación en Educación Superior, RED: Revista de Educación a Distancia, 58. Consultado el (dd/mm/aaaa) en http://www.um.es/ead/red/58/

Vértiz-Osores, Ricardo Iván, Pérez-Saavedra, Segundo, Faustino-Sánchez, Miguel Ángel, Vértiz-Osores, Jacinto Joaquín, \& Alain, Lineth. (2019). Information and Communication Technology in Primary School Students within the Framework of Inclusive Education at a Special Basic Education Center. Propósitos y Representaciones, 7(1), 83-94. https://dx.doi.org/10.20511/pyr2019.v7n1.266 